\title{
The Risk of Incident Mild Cognitive Impairment and Progression to Dementia Considering Mild Cognitive Impairment Subtypes
}

\author{
Tzeyu L. Michaud ${ }^{a, b} \quad$ Dejun Su ${ }^{a, b} \quad$ Mohammad Siahpush ${ }^{b}$ \\ Daniel L. Murman ${ }^{c}$ \\ ${ }^{a}$ Center for Reducing Health Disparities, College of Public Health, University of Nebraska \\ Medical Center, Omaha, NE, USA; ${ }^{b}$ Department of Health Promotion, Social and Behavioral \\ Health, College of Public Health, University of Nebraska Medical Center, Omaha, NE, USA; \\ 'Behavioral and Geriatric Neurology Program, Department of Neurological Sciences, \\ University of Nebraska Medical Center, Omaha, NE, USA
}

\section{Keywords}

Alzheimer disease - Mild cognitive impairment - Dementia progression · Predictors of cognitive decline $\cdot$ APOE $\varepsilon 4$

\begin{abstract}
Background: It remains unclear how demographic and clinical characteristics are related to the risk of incident mild cognitive impairment (MCI) by its subtypes. Moreover, the contribution of the subtypes of incident MCI to the progression to dementia remains puzzling. Methods: We used data collected by the National Alzheimer Coordinating Center. Our analysis sample included cognitively normal subjects at baseline. The associations were examined using competing-risks survival regression models and Cox proportional hazards models. Results: About $16.3 \%$ of subjects developed incident $\mathrm{MCI}$ of whom $15.8 \%$ progressed to Alzheimer disease (overall mean follow-up of 4.3 years). The risk of incident amnestic MCI (aMCI) was greater in subjects with 1 copy (subhazard ratio [SHR]: 1.23; 95\% CI: 1.00-1.50) or 2 copies (SHR: 2.14; 95\% CI: 1.49-3.05) of the APOE $\varepsilon 4$ allele than in those who had no $\varepsilon 4$ allele. Multiple-domain aMCI patients were more likely to progress to dementia than single-domain aMCI patients (hazard ratio: 2.14; 95\% CI: 1.28-3.58). Conclusions: Cognitively normal subjects with an APOE $\varepsilon 4$ allele had a higher likelihood of developing aMCI and the MCI subtype was associated with the dementia subtype. Our findings provide important information about practical indicators for the prediction of cognitive decline.


Michaud et al.: The Risk of Incident Mild Cognitive Impairment and Progression to Dementia Considering Mild Cognitive Impairment Subtypes

\section{Introduction}

Growing evidence has shown that the construct of mild cognitive impairment (MCI) can help researchers better understand the etiology of $\mathrm{MCI}$ and better predict the risk of progression from MCI to dementia, therefore facilitating early disease identification and disease management which, in turn, improves patients' outcomes [1-4]. MCI was initially conceptualized as a prodromal state of dementia disorder [5] or a transitional cognitive stage between normal cognitive aging and Alzheimer disease (AD) [5, 6] or dementia [4]. Most studies today further differentiate between amnestic MCI (aMCI) and nonamnestic MCI (naMCI) depending on whether or not memory is impaired, and many also classify these two entities into single-domain and multiple-domain MCI, depending on how many cognitive domains are impaired $[3,7]$. Moreover, a conceptual linkage between subtypes of MCI (aMCI vs. naMCI, either single- or multiple-domain) and different types of dementia has been proposed [3]; however, while aMCI is seen to be highly associated with progression to AD [1], other subtypes of MCI have been shown to present an inconsistent association with subsequent conversion to dementia.

Several studies have shown that MCI patients are at increased risk of progression to AD, with an annual conversion rate of $3-10 \%$ in the community settings and $10-15 \%$ in specialty clinics $[5,6,8,9]$. This discrepancy is due to the heterogeneous definition and description of MCI and different follow-up periods of studies $[10,11]$. However, not all subjects with MCI will develop AD or dementia (although they are still at greater risk than cognitively normal subjects) and some will remain stable or even return to normal cognition [12,13]. Furthermore, it is unclear whether subtypes of MCI have different prognoses for progression to dementia and which type and timing of dementia the subtypes predict. It is possible that all progressive dementias have their own predementia states [2]. Although not all studies have shown a strong connection between the subtype of MCI and a particular type of dementia into which it may progress, most researchers agree that subtypes of MCI have an influence on subsequent types of dementia diagnosed [14-17]. Therefore, it is crucial to understand the demographic and clinical predispositions of different subtypes of $\mathrm{MCI}$ and further examine how this insight may be linked to the subsequent progression to AD or other dementias.

Little is known regarding the relationship between the subtype of incident MCI and the subsequent progression to dementia in terms of the role of age at onset of MCI. Accordingly, the objectives of the confirmatory study, using a convenience sample from the National Alzheimer's Coordinating Center (NACC), were twofold: (1) to examine the characteristics of cognitively normal subjects who developed incident MCI by subtypes over time; and (2) to determine the association between subtypes of incident MCI and the progression to different types of dementia.

\section{Methods}

\section{Study Sample}

All data were obtained from the Uniform Data Set (UDS) of the NACC which is funded by the National Institute of Aging [18], downloaded on October 7, 2015. The dataset includes subjects with a range of cognitive characteristics, i.e. normal cognition, $\mathrm{MCI}$, and demented, and their demographic, clinical, and specimen data from 39 past and present Alzheimer Disease Centers (ADCs), which enroll and follow subjects with their own protocol. The UDS was collected via a standardized evaluation of subjects either during the office visit, home visit, or over the phone by a trained clinician or clinic personnel, getting the information needed either from the subjects themselves or their informants during the annual assessment. 
Michaud et al.: The Risk of Incident Mild Cognitive Impairment and Progression to Dementia Considering Mild Cognitive Impairment Subtypes

Written informed consent was obtained from all subjects and informants. Research using the NACC database was approved by the Institutional Review Board at the University of Washington. Because the recruitment objectives and referral methods vary greatly by each ADC, the NACC UDS is not a population-based sample and would thus not be appropriate to be applied to estimates for the general US population [19].

For this study, our analysis sample included subjects with normal cognition at baseline and had at least one follow-up evaluation. We excluded subjects who were impaired but had not MCI and those who were institutionalized or unable to live independently at baseline. In general, our analysis sample consisted of 8,691 subjects with normal cognition at baseline with an overall mean follow-up of 4.3-years.

\section{Diagnosis of MCI and Its Subtypes and Dementia}

We used NACC-derived variables to define individuals with incident $\mathrm{MCI}$ as subjects with normal cognition at the initial visit who, however, were diagnosed with MCI at the follow-up visit. This included those who reverted from MCI back to normal cognition. Moreover, the NACC database provided information about the subtypes of MCI diagnosis based on the framework suggested by Petersen et al. [3]. This allowed us to further classify incident MCI into 4 subtypes based on whether or not memory was impaired and whether or not only a single cognitive domain was impaired. These 4 subtypes are: single-domain aMCI (MCI with memory impaired only, aMCI-SD), multiple-domain aMCI (aMCI with other cognitive domains impaired, aMCI-MD), single-domain naMCI (MCI with a single nonmemory domain impaired, naMCI-SD), and multiple-domain naMCI (naMCI with multiple nonmemory domains impaired, naMCI-MD).

We further used NACC-derived variables to define subjects with first incident AD (probable and possible AD) or dementia as a primary diagnosis as subjects who had developed incident $\mathrm{MCI}$ and subsequently converted to $\mathrm{AD}$ or dementia at follow-up. The rationale here was to better capture the time from the onset of MCI to dementia. We further documented the type of dementia by the subtype of incident MCI.

\section{Other Characteristics}

We defined the age at onset of MCI or dementia as the age when the first MCI or dementia incidence occurred. The education level was measured as the number of years of schooling. The body mass index was categorized into 4 groups: $<18.5$ (underweight), 18.5-24.9 (normal weight), 25-29.9 (overweight), and $\geq 30$ (obesity) [20]. We summed the score of each task in the Functional Activities Questionnaire (FAQ) - with $0=$ normal, $1=$ has difficulty, but can finish by himself or herself, 2 = requires assistance, $3=$ dependent - to derive a total FAQ score (range 0-30) [21]. Similarly, we totalized the score in the Neuropsychiatric Inventory Questionnaire (NPI-Q), which was used to assess the severity for 12 neuropsychiatric disturbances common in dementia [22] - with $0=$ no disturbance, $1=$ mild, $2=$ moderate, and $3=$ severe - to derive the total NPI-Q score (range 0-36). Other covariates included age at baseline, sex, marital status, race, living situation, housing type, history of the use of Food and Drug Administration-approved AD medication, history of heart disease, APOE $\varepsilon 4$ genotype, history of first-degree family member with dementia, dependence level, Mini-Mental State Examination (MMSE; range 0-30) [23], global clinical dementia rating (CDR; range 0-3) [24], and CDR sum of box (CDR-SB; range 0-18) [25].

\section{Statistical Analysis}

We compared baseline demographic characteristics and clinical measurements by subtypes of incident MCI as well as incident MCI cases and those who remained stable using a nonparametric Kruskal-Wallis test for continuous variables and the Pearson $\chi^{2}$ test or 
Fisher exact test (in instances where cell numbers were small) for categorical variables. For the potential missing data issue, we documented the missing observations for each survival analysis.

\section{Risk of Incident MCI}

The definition of incident MCI was mentioned previously. We defined controls as subjects with normal cognition who had never been diagnosed with dementia at any visit.

Because we were interested in the relationship between baseline demographic and clinical characteristics and the risk of developing incident MCI, by subtype, we performed competing-risks survival regression models [26] to examine this relationship and calculated the covariate-adjusted subhazard ratio (SHR). With the assumption of independence between events, the risk set of this measure was constructed to include both subjects who had the competing event and subjects without any event [27]. We treated aMCI as the event of interest since it is highly associated with the conversion to AD-type dementia, and naMCI was considered as the competing event. Furthermore, we conducted additional analyses considering aMCI-SD as the event of interest and aMCI-MD, naMCI-SD, and naMCI-MD as competing events.

The onset of MCI was assumed to have taken place in the middle of each follow-up interval (average 1 year). This was operationalized by adding a time correction of 0.5 years to the time of first MCI diagnoses.

\section{Risk of Incident Dementia}

Survival analysis using Kaplan-Meier methods was applied to determine if the subtype of MCI was associated with the risk of dementia. We used log-rank tests to compare the risk of progression to dementia among subtypes of MCI. Cox proportional hazards models were used to evaluate the impact of demographic and clinical factors on the risk of progression. The proportional hazards assumption was assessed using the log-log plots of the survival function with Schoenfeld residuals [28]. The onset of dementia was assigned at the midpoint between the last assessment of MCI and the first-ever assessment of dementia. Similarly, we applied a 0.5-year correction to the time of conversion to dementia.

Finally, for those with incident MCI who had converted to dementia, we examined if there was an association between MCI subtypes and different types of dementia diagnosis (AD, vascular, Lewy bodies dementia, frontal, mixed, or other) using the Fisher exact test [17].

All analyses were conducted using Stata version 14 (StataCorp, College Station, TX, USA). Two-tailed $p$ values of less than 0.05 were considered significant.

\section{Results}

\section{Characteristics of the Sample}

Table 1 describes the demographic and clinical characteristics of the study sample. Among 8,691 subjects with normal cognition at baseline, 7,276 remained stable, and 1,415 (16.3\%) had developed incident MCI: 602 had aMCI-SD, 473 had aMCI-MD, 260 had naMCI$\mathrm{SD}$, and 80 had naMCI-MD, with a mean follow-up time of 3.06 years. The mean age of onset of $\mathrm{MCI}$ was 80 years regardless of the subtypes.

Among those who had developed incident MCI, the mean age at baseline was 76 years, $60 \%$ were female, $44 \%$ had received education at a high school level, $55 \%$ were married and were living with spouses, $82 \%$ were white, $61 \%$ were overweight, $85 \%$ were living in an apartment or house, 3\% used a Food and Drug Administration-approved AD medication, such as tacrine, donepezil, rivastigmine, galantamine, and memantine, 53\% had a first-degree 
Dementia

Cognitive Disorders

\begin{tabular}{l|l}
\hline DOI: $10.1159 / 000452486$ & $\begin{array}{l}\text { C } 2017 \text { The Author(s). Published by S. Karger AG, Basel } \\
\text { www.karger.com/dee }\end{array}$
\end{tabular}

Michaud et al.: The Risk of Incident Mild Cognitive Impairment and Progression to Dementia Considering Mild Cognitive Impairment Subtypes

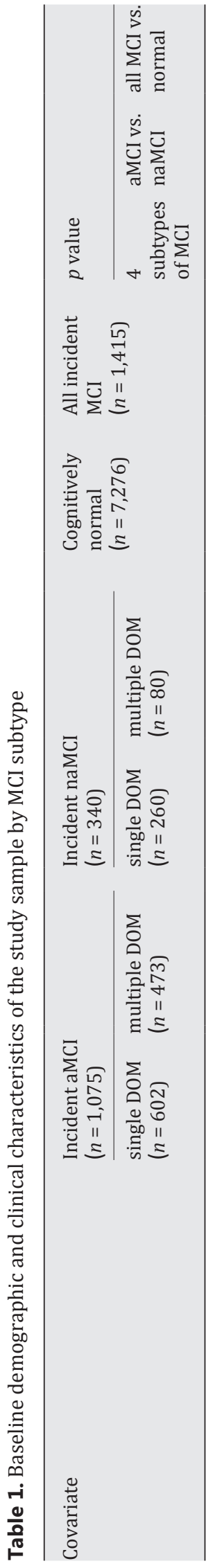

\begin{tabular}{|c|c|c|c|}
\hline 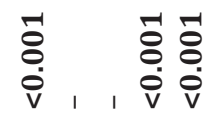 & 苂 & $\begin{array}{l}\tilde{g} \\
\dot{0}\end{array}$ & $\begin{array}{l}0 \\
\ddot{n} \\
0 \\
0\end{array}$ \\
\hline 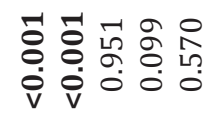 & $\begin{array}{l}\stackrel{m}{\Xi} \\
0 \\
0\end{array}$ & 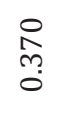 & $\begin{array}{l}\tilde{8} \\
\dot{0} \\
\dot{v}\end{array}$ \\
\hline 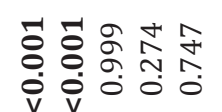 & $\begin{array}{l}\stackrel{0}{0} \\
\dot{0}\end{array}$ & $\begin{array}{l}\stackrel{m}{P} \\
\stackrel{+}{0} \\
0\end{array}$ & $\begin{array}{l}\tilde{8} \\
0 \\
0\end{array}$ \\
\hline
\end{tabular}

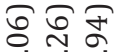

$0 \dot{0}$

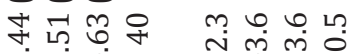

穴

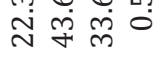

ํำ

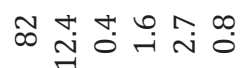

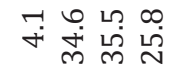

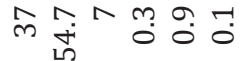

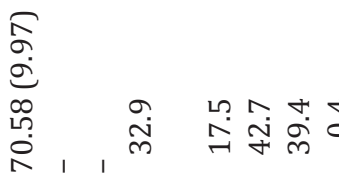

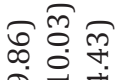

ف료

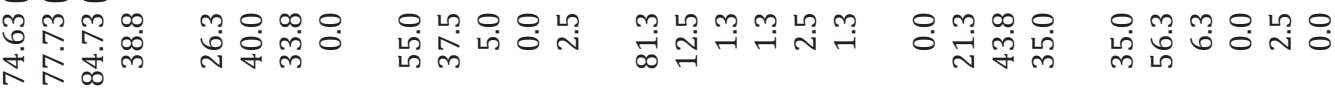


ळ $0 \dot{0}$

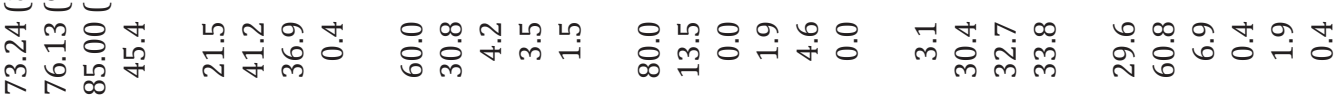

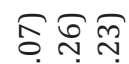

$\hat{\sigma} \sigma \tilde{\sigma}$

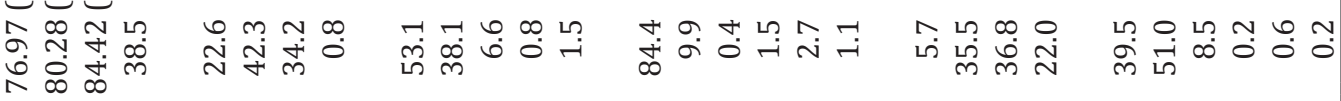
F⿱乛⿻

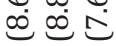

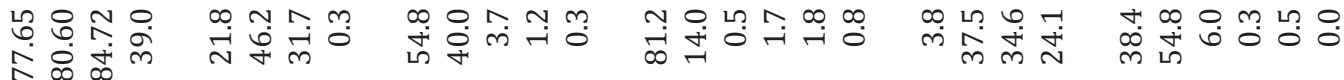
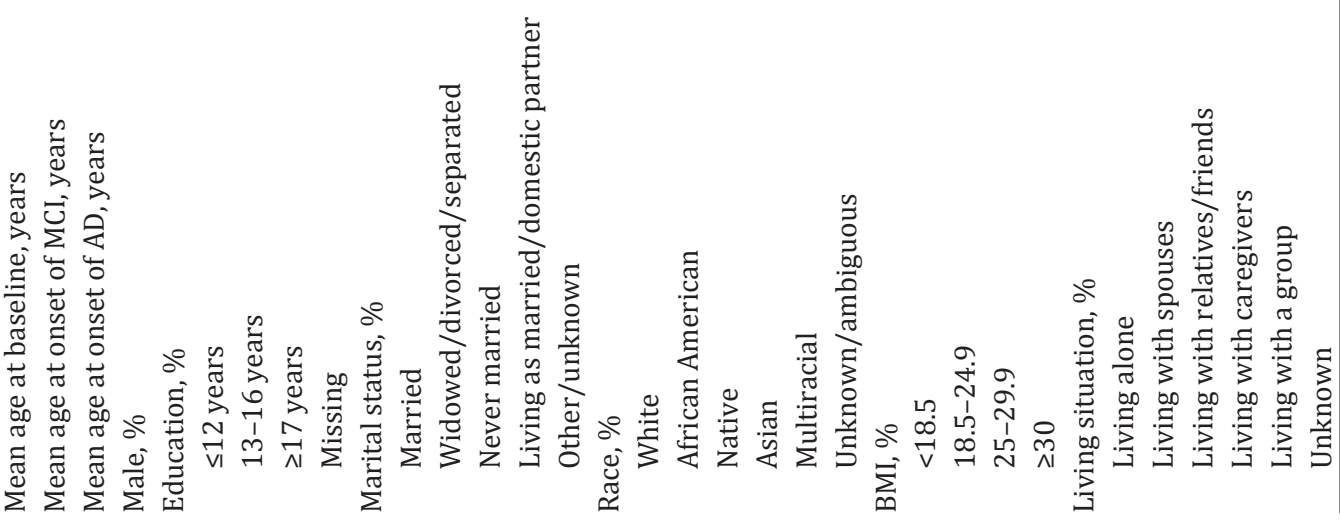
Dementia

Cognitive Disorders

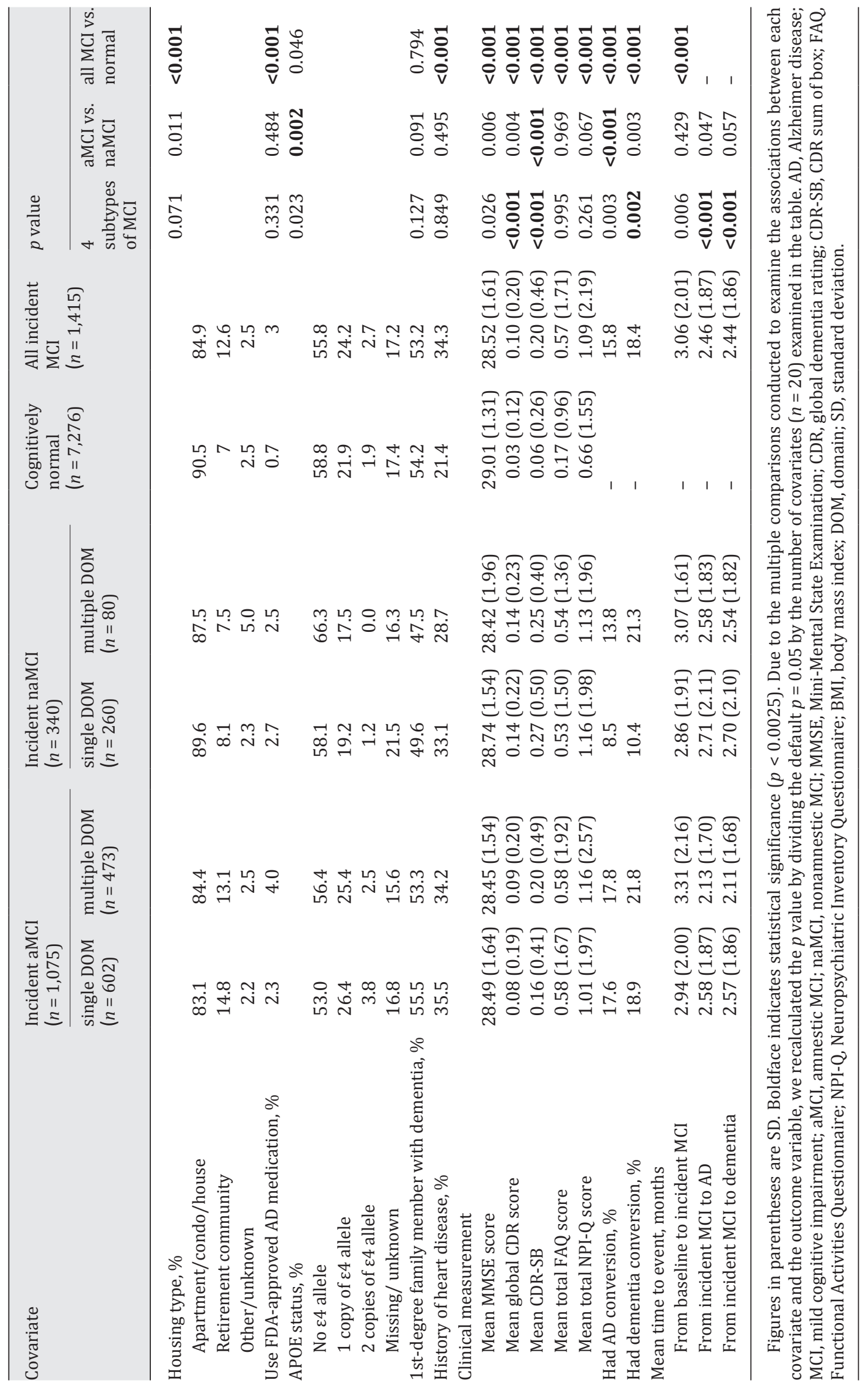

\begin{tabular}{l|l}
\hline DOI: $10.1159 / 000452486$ & C 2017 The Author(s). Published by S. Karger AG, Basel
\end{tabular} www.karger.com/dee

Michaud et al.: The Risk of Incident Mild Cognitive Impairment and Progression to Dementia Considering Mild Cognitive Impairment Subtypes

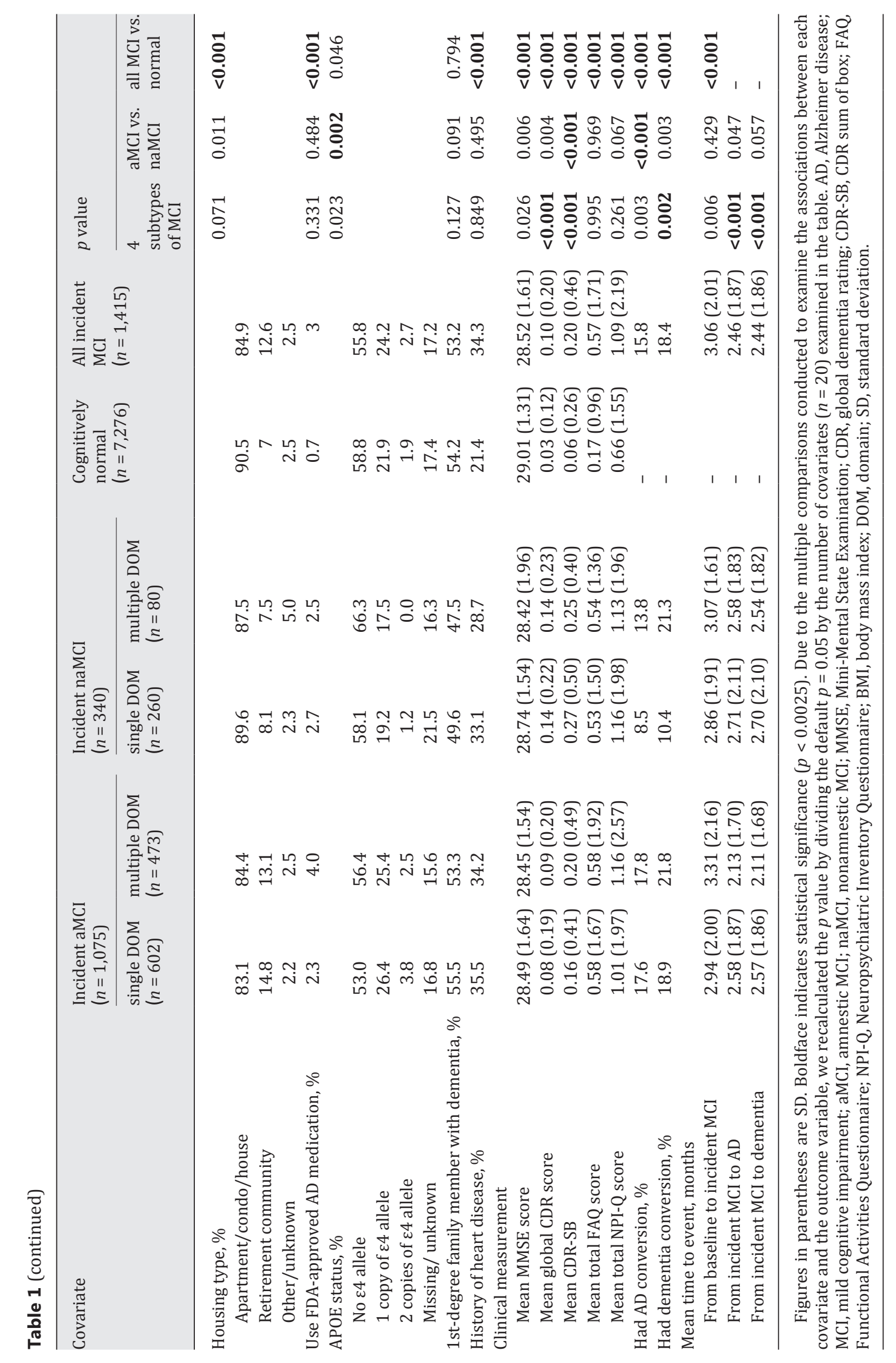


family member being diagnosed with dementia, $34 \%$ had experienced heart disease, and $27 \%$ carried the APOE $\varepsilon 4$ genotype.

We compared the baseline characteristics of the subjects by MCI subtypes (Table 1) and found significant differences in terms of age at baseline, age at onset of MCI, marital status, body mass index category, APOE $\varepsilon 4$ genotype, MMSE, CDR, CDR-SB, and whether or not they developed $\mathrm{AD}$ or dementia regardless the subtypes of $\mathrm{MCI}$.

\section{Risk of Incident MCI}

By MCI subtypes, the competing-risks survival regression results using aMCI as the event of interest showed that subjects who were older at baseline were associated with a $4 \%$ higher risk of developing aMCI among cognitively normal subjects (Table 2). Furthermore, the risk of incident aMCI was 23 or $114 \%$ greater in subjects with 1 copy or 2 copies of the APOE $\varepsilon 4$ allele. Of note, lower MMSE and CDR-SB scores were associated with a greater risk of incident aMCI (SHRs: 0.86 and 0.52, respectively), whereas subjects who were married or living with spouses were associated with a 46 or $45 \%$ risk reduction of developing aMCI compared to those who were widowed or divorced or living alone.

Moreover, Table 2 reports similar findings when using aMCI-SD as the event of interest. The risk of incident aMCI-SD was $41 \%$ greater in subjects with 1 copy of the APOE $\varepsilon 4$ allele than in those who had no $\varepsilon 4$ allele.

\section{Risk of Incident Dementia}

Of 1,415 incident MCI subjects, 223 (15.8\%) had developed AD of whom 106 had aMCI-SD, 84 had aMCI-MD, 22 had naMCI-SD, and 11 had naMCI-MD, with a mean follow-up time of 2.46 years. The mean age of onset of $\mathrm{AD}$ was 85 years.

Table 3 shows that the subtype of MCI was not a confounder to the risk of AD among subjects with incident MCI with either 4 subtypes or 2 subtypes. In addition, Table 3 presents similar findings when examining the association between the characteristics and the risk of dementia. Most importantly, subjects with aMCI-MD had a higher likelihood of converting to dementia than subjects with aMCI-SD (hazard ratio: 2.14; 95\% CI: 1.28-3.58).

Without adjusting for baseline characteristics, the result of the log-rank test indicated the risk of progression from incident MCI to AD to be significantly differed by the subtype of MCI $(p<0.001)$, either among the 4 subtypes or between 2 subtypes (not shown). Subjects with naMCI-SD had the highest AD-free survival, then subjects with naMCI-MD, subjects with aMCI-SD, and subjects with aMCI-MD in a descending order (Fig. 1a). However, different results were observed after adjusting for the covariates in the Cox proportional hazards models. Subjects with MCI-SD (either aMCI or naMCI) were those with the higher AD-free survival than subjects with MCI-MD. Subjects with aMCI-MD still had the lowest AD-free survival. In addition, we found a similar pattern for the adjusted survival when considering all types of dementia as a whole (Fig. 1b).

\section{Association between MCI Subtypes and Types of Dementia Diagnosis}

Table 4 reports the frequency of reversion to normal cognition, and dementia conversion among incident MCI patients. A total of 350 (25\%) subjects with incident MCI had reverted to normal cognition during the follow-up. Moreover, among those who had progressed to dementia, 44\% had aMCI-SD, 40\% had aMCI-MD, 10\% had naMCI-SD and 7\% had naMCI-MD. There were significant differences between the type of dementia and the subtype of incident $\operatorname{MCI}(p=0.014)$. 
Table 2. Hazard and subhazard ratios of incident MCI among cognitively normal subjects

\begin{tabular}{|c|c|c|c|c|c|c|}
\hline \multirow[t]{2}{*}{ Covariate/event of interest } & \multicolumn{2}{|c|}{ Incident MCI } & \multicolumn{2}{|l|}{$\mathrm{aMCI}$} & \multicolumn{2}{|c|}{ aMCI-SD } \\
\hline & $\mathrm{HR}^{\mathrm{a}}$ & $95 \% \mathrm{CI}^{\mathrm{c}}$ & $\mathrm{SHR}^{\mathrm{b}}$ & $95 \% \mathrm{CI}^{\mathrm{c}}$ & $\mathrm{SHR}^{\mathrm{b}}$ & $95 \% \mathrm{CI}^{\mathrm{c}}$ \\
\hline Age at baseline & 1.03 & $1.02-1.04$ & 1.04 & 1.03-1.05 & 1.04 & 1.03-1.06 \\
\hline Sex $($ female $=0)$ & 0.96 & $0.8-1.16$ & 1.06 & $0.86-1.30$ & 1.31 & 1.01-1.71 \\
\hline \multicolumn{7}{|l|}{ Education $(\leq 12=0)$} \\
\hline $13-16$ years & 1.29 & 1.05-1.95 & 1.18 & $0.93-1.49$ & 1.39 & $1.03-1.8$ \\
\hline$\geq 17$ years & 1.07 & $0.85-1.35$ & 0.99 & $0.77-1.29$ & 1.06 & $0.75-1.50$ \\
\hline \multicolumn{7}{|l|}{ Marital status (married = 0) } \\
\hline Widowed/divorced/separated & 0.64 & $0.42-0.98$ & 0.54 & $0.34-0.84$ & 0.71 & $0.37-1.38$ \\
\hline Never married & 0.83 & $0.49-1.41$ & 0.62 & $0.34-1.13$ & 0.47 & $0.19-1.16$ \\
\hline Living as married/ domestic partner & 1.65 & $0.92-2.96$ & 1.21 & $0.57-2.58$ & 1.79 & $0.75-4.28$ \\
\hline \multicolumn{7}{|l|}{ Race $($ White $=0)$} \\
\hline African American & 1.16 & $0.91-1.49$ & 1.13 & $0.85-1.51$ & 1.06 & $0.73-1.55$ \\
\hline Native & 1.48 & $0.37-5.97$ & 2.15 & $0.93-4.99$ & 1.58 & $0.31-7.99$ \\
\hline Asian & 0.68 & $0.32-1.44$ & 0.54 & $0.27-1.09$ & 0.61 & $0.21-1.81$ \\
\hline Multiracial & 0.85 & $0.53-1.37$ & 0.64 & $0.35-1.19$ & 0.45 & $0.18-1.11$ \\
\hline \multicolumn{7}{|l|}{ BMI $(<18.5=0)$} \\
\hline $18.5-24.9$ & 1.12 & $0.72-1.74$ & 1.11 & $0.70-1.76$ & 2.75 & 1.14-6.64 \\
\hline $25-29.9$ & 1.08 & $0.69-1.69$ & 1.02 & $0.64-1.62$ & 2.62 & 1.08-6.35 \\
\hline$\geq 30$ & 1.01 & $0.64-1.61$ & 0.82 & $0.50-1.36$ & 2.14 & $0.86-5.34$ \\
\hline \multicolumn{7}{|l|}{ Living situation (living alone $=0$ ) } \\
\hline Living with spouses & 0.65 & $0.42-1.00$ & 0.55 & $0.35-0.86$ & 0.86 & $0.44-1.69$ \\
\hline Living with relatives/friends & 0.94 & $0.70-1.28$ & 0.93 & $0.66-1.31$ & 0.85 & $0.53-1.35$ \\
\hline Living with caregivers & 0.70 & $0.17-2.88$ & 1.12 & $0.31-4.05$ & - & - \\
\hline Living with a group & 0.50 & $0.07-3.60$ & 0.00 & - & - & - \\
\hline \multicolumn{7}{|l|}{ Housing type (apartment/condo $=0$ ) } \\
\hline Retirement community & 0.85 & $0.67-1.09$ & 0.95 & $0.73-1.24$ & 0.92 & $0.65-1.29$ \\
\hline Use of FDA-approved AD medication & 1.52 & $1.03-2.23$ & 1.27 & $0.80-2.03$ & 0.73 & $0.39-1.37$ \\
\hline History of heart disease & 0.92 & $0.77-1.09$ & 1.04 & $0.86-1.26$ & 0.98 & $0.76-1.26$ \\
\hline \multicolumn{7}{|l|}{ APOE status (number of $\varepsilon 4$ allele $=0$ ) } \\
\hline 1 copy of $\varepsilon 4$ allele & 1.13 & $0.95-1.34$ & 1.23 & $1.00-1.50$ & 1.41 & $1.10-1.80$ \\
\hline 2 copies of $\varepsilon 4$ allele & 1.53 & $0.99-2.37$ & 2.14 & 1.49-3.05 & 1.62 & $0.93-2.83$ \\
\hline 1st-degree family member with dementia & 0.95 & $0.81-1.11$ & 1.11 & $0.92-1.34$ & 1.13 & $0.89-1.43$ \\
\hline \multicolumn{7}{|l|}{ Clinical outcomes } \\
\hline MMSE & 0.86 & $0.83-0.90$ & 0.86 & $0.82-0.90$ & 0.88 & $0.83-0.94$ \\
\hline CDR sum of box & 0.68 & $0.59-0.79$ & 0.52 & $0.41-0.65$ & 0.29 & 0.21-0.39 \\
\hline Total FAQ scores & 0.98 & 0.95-1.01 & 0.99 & $0.94-1.04$ & 1.02 & $0.97-1.07$ \\
\hline Total NPI-Q scores & 1.00 & $0.96-1.03$ & 0.98 & $0.94-1.03$ & 0.98 & $0.92-1.04$ \\
\hline
\end{tabular}

Total number of subjects who were cognitively normal at baseline and who did not develop incident MCI were 7,276. Missing data was low to moderate, with education (28 subjects), marital status (76), race (29), living situation (23), housing type (182), use of FDA-approved AD medication (156), history of heart disease (56), APOE status (1,265), and 1st-degree family member with dementia (151), MMSE (230), total FAQ scores $(1,236)$, and total NPI-Q scores (483). Total number of subjects who were cognitively normal at baseline and who developed incident MCI were 1,415. Missing data was low, with education (7 subjects), marital status (15), race (11), living situation (2), housing type (35), use of FDA-approved AD medication (32), history of heart disease (7), APOE status (244), and 1st-degree family member with dementia (31), MMSE (93), total FAQ scores (329), and total NPI-Q scores (150). MCI, mild cognitive impairment; aMCI, amnestic MCI; aMCI-SD, aMCI-single domain; MMSE, Mini-Mental State Examination; CDR-SB, global dementia rating-sum ofbox; FAQ, Functional Activities Questionnaire; NPI-Q, Neuropsychiatric Inventory Questionnaire; BMI, body mass index; HR, hazard ratio; SHR, sub-hazard ratio.

${ }^{\mathrm{a}}$ HR was estimated by Cox proportional hazards models. ${ }^{\mathrm{b}}$ SHR was computed from the competing-risk survival regression model. ${ }^{\mathrm{c}}$ Text in bold represents a statistical significance. 
Michaud et al.: The Risk of Incident Mild Cognitive Impairment and Progression to Dementia Considering Mild Cognitive Impairment Subtypes

Table 3. Hazard ratios of progressing from incident MCI to dementia by demographic and clinical characteristics

\begin{tabular}{|c|c|c|c|c|}
\hline \multirow[t]{2}{*}{ Covariate } & \multicolumn{2}{|l|}{$\mathrm{AD}$} & \multicolumn{2}{|c|}{ Dementia } \\
\hline & HR & $95 \% \mathrm{CI}^{\mathrm{a}}$ & HR & $95 \% \mathrm{CI}^{\mathrm{a}}$ \\
\hline Age at baseline & 0.90 & $0.77-1.04$ & 0.91 & $0.79-1.04$ \\
\hline Age at onset of MCI & 1.17 & 1.01-1.35 & 1.15 & $1.00-1.32$ \\
\hline Sex (female $=0$ ) & 1.14 & $0.63-2.07$ & 0.98 & $0.55-1.76$ \\
\hline \multicolumn{5}{|l|}{ Education $(\leq 12=0)$} \\
\hline $13-16$ years & 0.56 & $0.32-0.98$ & 0.48 & $0.29-0.81$ \\
\hline$\geq 17$ years & 0.29 & $0.14-0.59$ & 0.20 & $0.10-0.42$ \\
\hline \multicolumn{5}{|l|}{ Marital status (married $=0$ ) } \\
\hline Widowed/divorced/separated & 0.72 & $0.20-2.58$ & 0.30 & $0.09-1.02$ \\
\hline Never married & 2.85 & $0.68-12.05$ & 0.98 & $0.23-4.14$ \\
\hline Living as married/ domestic partner & 4.11 & $1.25-13.46$ & 2.54 & $0.67-9.67$ \\
\hline \multicolumn{5}{|l|}{ Race $($ White $=0)$} \\
\hline African American & 1.64 & $0.69-3.87$ & 1.39 & $0.60-3.22$ \\
\hline Native & 1.00 & - & & \\
\hline Asian & 1.17 & $0.14-9.98$ & 1.19 & $0.14-9.97$ \\
\hline Multiracial & 2.50 & $0.72-8.65$ & 2.35 & $0.69-7.95$ \\
\hline \multicolumn{5}{|l|}{ BMI $(<18.5=0)$} \\
\hline $18.5-24.9$ & 2.12 & $0.72-6.19$ & 1.31 & $0.45-3.82$ \\
\hline $25-29.9$ & 1.46 & $0.50-4.28$ & 0.87 & $0.30-2.58$ \\
\hline$\geq 30$ & 1.36 & $0.43-4.25$ & 0.96 & $0.31-2.91$ \\
\hline \multicolumn{5}{|l|}{ Living situation (living alone $=0$ ) } \\
\hline Living with spouses & 0.90 & $0.26-3.19$ & 0.35 & $0.10-1.18$ \\
\hline Living with relatives/friends & 0.58 & $0.25-1.38$ & 0.62 & $0.27-1.41$ \\
\hline Living with caregivers & 1.06 & $0.31-3.63$ & 2.44 & $0.68-8.71$ \\
\hline Living with a group & 2.94 & $0.96-8.97$ & 3.91 & $1.40-10.93$ \\
\hline \multicolumn{5}{|l|}{ Housing type $($ apartment $/$ condo $=0$ ) } \\
\hline Retirement community & 0.83 & $0.42-1.65$ & 0.66 & $0.33-1.33$ \\
\hline Assisted living & 0.38 & $0.15-0.93$ & 0.19 & $0.07-0.53$ \\
\hline Nursing home & 0.15 & $0.01-1.79$ & 0.18 & $0.02-1.72$ \\
\hline Use of FDA-approved AD medication & 2.46 & $1.45-4.17$ & 2.38 & $1.40-4.03$ \\
\hline History of heart disease & 1.00 & $0.63-1.60$ & 1.01 & $0.63-1.62$ \\
\hline \multicolumn{5}{|l|}{ APOE status (number of $\varepsilon 4$ allele $=0$ ) } \\
\hline 1 copy of $\varepsilon 4$ allele & 1.48 & $0.91-2.41$ & 1.58 & $0.99-2.51$ \\
\hline 2 copies of $\varepsilon 4$ allele & 1.31 & $0.29-5.99$ & 1.36 & $0.31-6.09$ \\
\hline 1st-degree family member with dementia & 1.34 & $0.81-2.22$ & 1.58 & $0.97-2.58$ \\
\hline \multicolumn{5}{|l|}{ Clinical outcomes } \\
\hline MMSE & 0.99 & $0.90-1.07$ & 0.96 & $0.88-1.04$ \\
\hline Global CDR & 0.38 & $0.11-1.25$ & 0.43 & $0.13-1.36$ \\
\hline CDR sum of box & 1.41 & 1.11-1.79 & 1.44 & $1.12-1.84$ \\
\hline Total FAQ scores & 1.02 & $0.98-1.07$ & 1.02 & $0.97-1.07$ \\
\hline Total NPI-Q scores & 1.00 & $0.93-1.08$ & 0.99 & $0.92-1.07$ \\
\hline \multicolumn{5}{|l|}{ Independence level (able to live independently $=0$ ) } \\
\hline Requires some assistance with complex activities & 3.11 & $1.72-5.61$ & 2.73 & 1.51-4.94 \\
\hline Requires some assistance with basic activities & 2.63 & $1.08-6.38$ & 1.81 & $0.73-4.48$ \\
\hline Completely dependent & 0.67 & $0.08-5.27$ & 10.64 & $1.06-107.10$ \\
\hline \multicolumn{5}{|l|}{ MCI type $(\mathrm{aMCI}-\mathrm{SD}=0)$} \\
\hline aMCI-MD & 1.52 & $0.90-2.56$ & 2.14 & $1.28-3.58$ \\
\hline naMCI-SD & 0.97 & $0.42-2.22$ & 0.86 & $0.38-1.97$ \\
\hline naMCI-MD & 1.64 & $0.59-4.53$ & 1.65 & $0.55-4.95$ \\
\hline
\end{tabular}

Total number of subjects who developed incident MCI at follow-up and subsequently did not progress to AD were 1,192. Missing data was low, with education (6 subjects), marital status (12), race (10), living situation (2), housing type (31), use of FDA-approved AD medication (19), history of heart disease (4), APOE status (214), and 1st-degree family member with dementia (19), MMSE (78), total FAQ scores (283), and total NPI-Q scores (119). Total number of subjects who developed incident MCI at follow-up and who subsequently progressed to AD were 223. Missing data was low, with education (1 subjects), marital status (3), race (1), housing type (4), use of FDAapproved AD medication (13), history of heart disease (3), APOE status (30), and 1st-degree family member with dementia (12), MMSE (15), total FAQ scores (46), and total NPI-Q scores (31). MCI, mild cognitive impairment; aMCI-SD, amnestic MCI-single domain; aMCI-MD, aMCI-multiple domain; naMCI-SD, nonamnestic MCI-single domain; naMCI-MD, nonamnestic MCI-multiple domain; AD, Alzheimer's disease; MMSE, Mini-Mental State Examination; CDR, global dementia rating; CDR-SB, CDR-sum of box; FAQ, Functional Activities Questionnaire; NPI-Q, Neuropsychiatric Inventory Questionnaire; BMI, body mass index; HR, hazard ratio. ${ }^{a}$ Text in bold represents a statistical significance. For both models, we did not find that the proportional hazard assumption was violated. 
Dementia

and Geriatric
Dement Geriatr Cogn Disord Extra

\begin{tabular}{|l|l|l|}
\hline DOI: $10.1159 / 000452486$ & C 2017 The Author(s). Published by S. Karger AG, Basel
\end{tabular} www.karger.com/dee

Michaud et al.: The Risk of Incident Mild Cognitive Impairment and Progression to Dementia Considering Mild Cognitive Impairment Subtypes

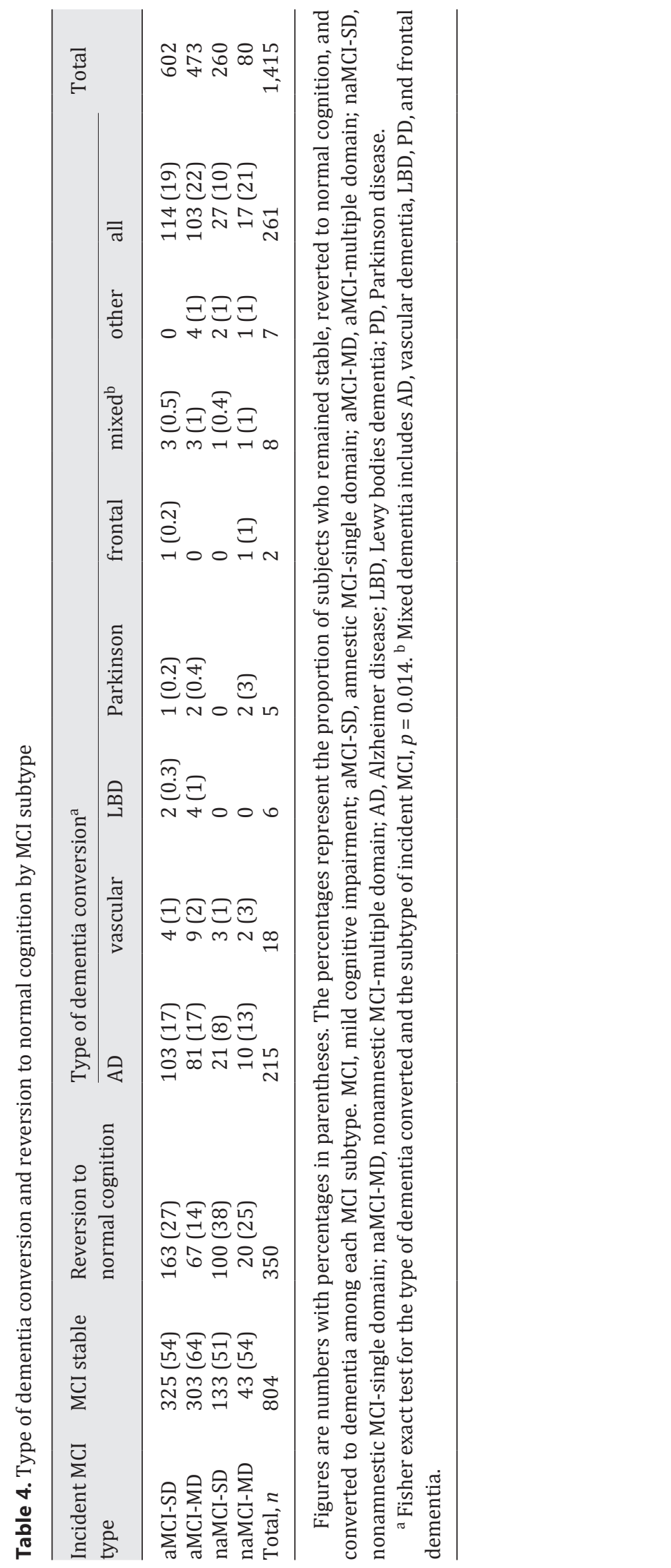




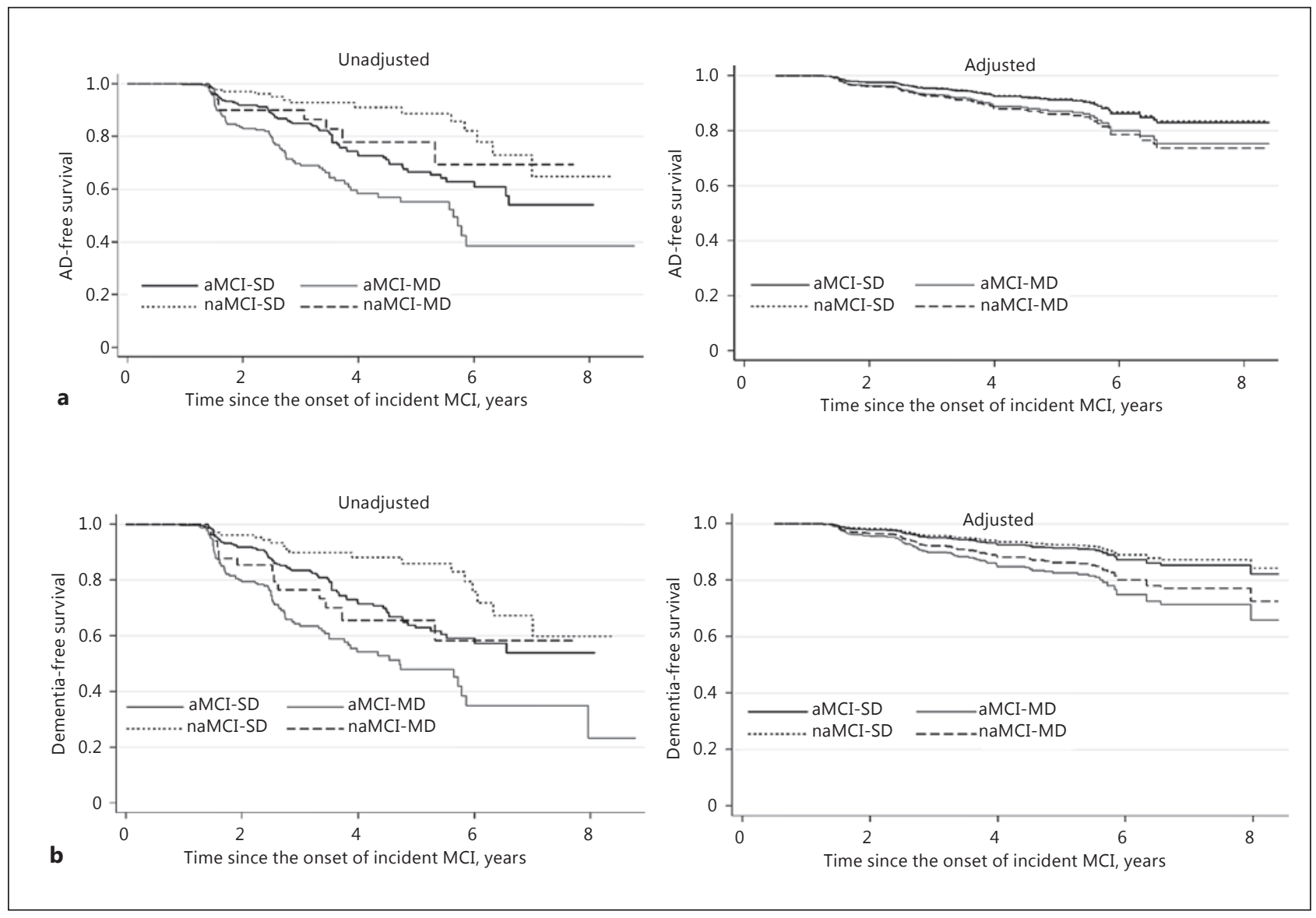

Fig. 1. Time to dementia conversion by MCI subtype. a AD. b Dementia. AD, Alzheimer disease.

\section{Discussion}

As the association between subtype of incident $\mathrm{MCI}$ and progression to $\mathrm{AD}$ or dementia remains unclear, this study sought to investigate this relationship. We did not find an association between MCI subtypes and the risk of $\mathrm{AD}$ after adjusting for other covariates. However, subjects with aMCI and impaired in multiple domains were more likely to convert to dementia than subjects with aMCI and impaired in a single domain. Not surprisingly, impaired in multiple cognitive domains is conceptualized to represent a more advanced disease stage than single-domain impairment, and patients at this stage are considered to be more likely to develop dementia $[3,5,13]$.

Corroborating studies by Pankratz et al. [29] and Knopman et al. [30], our results show that cognitively normal subjects with the APOE $\varepsilon 4$ allele were not only more likely to develop either aMCI or aMCI-SD, there was also a potential dose effect of the $\varepsilon 4$ genotype. The SHR of developing incident aMCI for those with 2 copies of the $\varepsilon 4$ allele was greater than for those with 1 copy. This finding could contribute to the utility of stratifying cognitively normal subjects into different risk levels of developing aMCI according to the presence of the APOE $\varepsilon 4$ genotype.

Our results showed that age at baseline was a risk factor for developing incident MCI as reported by Solfrizzi et al. [31], but was not associated with the risk of progressing from 
incident $\mathrm{MCI}$ to $\mathrm{AD}$, even though it was considered as an important predictor in previous studies $[15,32,33]$. This finding may relate to the relatively younger population in our study (mean age of 71 years at baseline) or the fact that previous studies were not able to adjust for the duration of MCI in their analysis on the risk of AD since they included subjects with an MCI diagnosis at baseline without the information of age at onset of MCI. It was speculated that it may take the same amount of time to progress from MCI to AD regardless of age [34]. In the bivariate analysis (Table 1), we found a significant difference in terms of conversion time from incident MCI to AD considering the 4 subtypes of MCI but a nonsignificant difference considering the 2 subtypes (aMCI vs. naMCI). To what extent the duration of MCI along with its subtypes may affect the conversion to AD remains to be examined further.

It is also likely that factors associated with the risk of incident MCI may be in turn relevant to the risk of progressing to $\mathrm{AD}$ due to the conceptual linkage between subtypes of $\mathrm{MCI}$ and different types of dementia [3], especially the association between aMCI and AD conversion. Numerous studies showed that the association of the APOE $\varepsilon 4$ allele with subsequent risk of AD [35] has been well established [36, 37]. However, our results demonstrated that this effect may be mediated by the adjustment for the duration of $\mathrm{MCI}$ [30]. The present study confirmed a significant association between the APOE $\varepsilon 4$ genotype and the risk of aMCI but disagreed that this factor was also associated with the progression from incident MCI to AD afterwards. It is likely that the APOE $\varepsilon 4$ allele initiates the AD process and makes clinical symptoms more likely to manifest; and the APOE effect on the disease progression diminishes once a diagnosis of $\mathrm{AD}$ is made. Consequently, the time to AD might not be influenced by APOE for subjects who had already been identified as having MCI, meaning the APOE effect was accounted for in the earlier disease stage, as suggested by our findings. Considering the substantial conflict in the literature regarding the effect of $\mathrm{APOE} \varepsilon 4$ on the progression from $\mathrm{MCI}$ to $\mathrm{AD}[34,36,38,39]$ and the few studies on the duration of $\mathrm{MCI}$, our findings add a different insight into the existing literature.

Our annual conversion rate from incident MCI to AD (6.4\%) was not deviant from what has been found in the literature (3-10\% in the community settings and $10-15 \%$ in specialty clinics) $[5,6,8,9]$, as well as the rate of $2.5 \%$ for cognitively normal subjects at baseline who developed dementia without an MCI diagnosis during the follow-up (1-2\% in the general population per year [5]). Most importantly, our study sought to elucidate the mean time from incident $\mathrm{MCI}$ to $\mathrm{AD}$, and thus we excluded subjects who were diagnosed as having MCI at baseline to account for the timing of the onset of MCI. Accordingly, it is possible that it takes more time to progress to $\mathrm{AD}$ from incident $\mathrm{MCI}$ in our case than in studies that included all $\mathrm{MCI}$ subjects at study baseline (regardless of their MCI duration); the shorter time to AD could result in higher conversion rates. Indeed, the annual conversion rate of AD increased from 6.4 to $9 \%$ after we included subjects who were diagnosed as having MCI at baseline in our analysis.

We found that the MCI subtype was associated with dementia diagnosis and AD was the most common type of dementia at follow-up among all 4 subtypes of MCI. This was similar to what Busse et al. [14] (except naMCI-MD), Jungwirth et al. [16] (only aMCI showed an increased risk for one particular type of dementia, namely AD), and Yaffe et al. [17] have found. They concluded that subtypes of MCI have a major influence on subsequent types of dementia diagnosis. Yet, we did not find an association between subtypes of MCI and the risk of $\mathrm{AD}$ after adjusting for other covariates, even though $76 \%$ of subjects with incident MCI had aMCI in our case. However, patients with aMCI-MD were more likely to progress to dementia after adjusting for other covariates than patients with aMCI-SD; this is possibly due to the sample size $(22 \%$ of aMCI-MD progressed to dementia compared to $17 \%$ who progressed to $\mathrm{AD}$ ) or the unstable diagnosis of MCI-SD (31\% reversed to normal cognition) as shown in the literature [40]. Of note, the $25 \%$ reversion rate from incident $\mathrm{MCI}$ to normal cognition was 
similar to estimates in the population-based studies [15, 40], which reported 29 and 31\% reversion rates.

We acknowledge some limitations. First, we may underestimate the amount of subjects who developed incident MCI or progressed to dementia from incident MCI, because we were notable to account for subjects who were lost to follow-up due to advanced disease progression or mortality. Hypothetically, subjects could have discontinued the study due to cognitive decline, functional impairment, or death. Second, for the survival analysis on the risk of incident $\mathrm{MCI}$, although about $17.4 \%$ of APOE $\varepsilon 4$ status was unknown or missing, our findings remain robust because $84 \%$ of these missing values appeared in subjects with normal cognition who did not develop incident MCI at follow-up. Furthermore, the sample size for the survival analyses was still considered sufficiently large even after excluding the observations with missing APOE information $(n=7,182)$. Third, because the gold standard of AD diagnosis is through the confirmation at autopsy, the inherent issue of clinical AD misdiagnosis remains intractable based on the current scientific advancement. By only including subjects with incident $\mathrm{MCI}$, progressed from normal cognition at baseline, in the analysis of risk of $\mathrm{AD}$ and the underdiagnosed or underreported issue of AD cases [41], diagnostic uncertainty should not have a considerable impact on our conclusions. Fourth, statistical significance was found in terms of clinical measurements (MMSE, CDR, CDR-SB, FAQ, and NPI-Q) between cognitively normal subjects who had developed MCI and those who remained intact. However, the observed significance may have resulted from a sufficiently large sample in our study and the effect size of each measurement was negligible based on the own operationalization, such as baseline MMSE scores of 28.52 versus 29.01, representing a half-point difference in terms of the 30-point scale. Fifth, although each ADC enrolls its subjects according to its own protocol, the UDS collection is administered using a standard protocol, which may reduce heterogeneity. The exclusion of individuals who were institutionalized, unable to live independently, or impaired but not having MCI at baseline from our analysis sample also calls for caution before our findings can be generalized to the elderly population in general. Lastly, although the NACC database is not a population-based study, and thus may not be ideal for conducting studies that investigate the risk profiles of incident $\mathrm{MCI}$ or incident dementia, this dataset still enriches our understanding with the detailed nation-wide information it encompassed, such as the subtypes of MCI and detailed dementia diagnosis, social relationship and so on. It shed some new light on the demographic and clinical predispositions of incident MCI and how the MCI subtype is related to the risk of incident dementia.

In conclusion, our study indicates that demographic and clinical characteristics can predict incident MCI and subsequent progression to dementia. Subjects with an APOE $\varepsilon 4$ allele had a higher likelihood of developing aMCI but not a higher risk of progressing from incident MCI to AD. In those who progressed from incident MCI to dementia, MCI subtype was significantly associated with dementia subtype and time to the onset of dementia. Our findings provide important information about practical indicators for the prediction of cognitive decline and might be applicable in the primary care setting.

\section{Acknowledgements}

Data used in the preparation of this article were obtained from the NACC. The NACC database is funded by NIA/NIH Grant U01 AG016976. NACC data are contributed by the NIAfunded ADCs: P30 AG019610 (PI Eric Reiman, MD), P30 AG013846 (PI Neil Kowall, MD), P50 AG008702 (PI Scott Small, MD), P50 AG025688 (PI Allan Levey, MD, PhD), P50 AG047266 (PI Todd Golde, MD, PhD), P30 AG010133 (PI Andrew Saykin, PsyD), P50 AG005146 (PI Marilyn Albert, PhD), P50 AG005134 (PI Bradley Hyman, MD, PhD), P50 AG016574 (PI Ronald 
Petersen, MD, PhD), P50 AG005138 (PI Mary Sano, PhD), P30 AG008051 (PI Steven Ferris, PhD), P30 AG013854 (PI M. Marsel Mesulam, MD), P30 AG008017 (PI Jeffrey Kaye, MD), P30 AG010161 (PI David Bennett, MD), P50 AG047366 (PI Victor Henderson, MD, MS), P30 AG010129 (PI Charles DeCarli, MD), P50 AG016573 (PI Frank LaFerla, PhD), P50 AG016570 (PI Marie-Francoise Chesselet, MD, PhD), P50 AG005131 (PI Douglas Galasko, MD), P50 AG023501 (PI Bruce Miller, MD), P30 AG035982 (PI Russell Swerdlow, MD) , P30 AG028383 (PI Linda Van Eldik, PhD), P30 AG010124 (PI John Trojanowski, MD, PhD), P50 AG005133 (PI Oscar Lopez, MD), P50 AG005142 (PI Helena Chui, MD), P30 AG012300 (PI Roger Rosenberg, MD), P50 AG005136 (PI Thomas Montine, MD, PhD), P50 AG033514 (PI Sanjay Asthana, MD, FRCP), P50 AG005681 (PI John Morris, MD), and P50 AG047270 (PI Stephen Strittmatter, MD, $\mathrm{PhD})$.

\section{Disclosure Statement}

The authors report no conflicts of interest related to the manuscript.

\section{References}

1 Albert MS, DeKosky ST, Dickson D, Dubois B, Feldman HH, Fox NC, Gamst A, Holtzman DM, Jagust WJ, Petersen RC: The diagnosis of mild cognitive impairment due to Alzheimer's disease: recommendations from the National Institute on Aging-Alzheimer's Association workgroups on diagnostic guidelines for Alzheimer's disease. Alzheimers Dement 2011;7:270-279.

-2 Gauthier S, Reisberg B, Zaudig M, Petersen RC, Ritchie K, Broich K, Belleville S, Brodaty H, Bennett D, Chertkow H: Mild cognitive impairment. Lancet 2006;367:1262-1270.

-3 Petersen RC, Morris JC: Mild cognitive impairment as a clinical entity and treatment target. Arch Neurol 2005;62:1160-1163.

4 Flicker C, Ferris SH, Reisberg B: Mild cognitive impairment in the elderly predictors of dementia. Neurology 1991;41:1006-1006.

5 Petersen RC, Smith GE, Waring SC, Ivnik RJ, Tangalos EG, Kokmen E: Mild cognitive impairment: clinical characterization and outcome. Arch Neurol 1999;56:303-308.

-6 Petersen RC, Doody R, Kurz A, Mohs RC, Morris JC, Rabins PV, Ritchie K, Rossor M, Thal L, Winblad B: Current concepts in mild cognitive impairment. Arch Neurol 2001;58:1985-1992.

-7 Hughes TF, Snitz BE, Ganguli M: Should mild cognitive impairment be subtyped? Curr Opin Psychiatry 2011;24: 237-242.

-8 Farias ST, Mungas D, Reed BR, Harvey D, DeCarli C: Progression of mild cognitive impairment to dementia in clinic- vs community-based cohorts. Arch Neurol 2009;66:1151-1157.

-9 Bruscoli M, Lovestone S: Is MCI really just early dementia? A systematic review of conversion studies. Int Psychogeriatr 2004;16:129-140.

10 Vemuri P, Wiste HJ, Weigand SD, Shaw LM, Trojanowski JQ, Weiner MW, Knopman DS, Petersen RC, Jack CR: MRI and CSF biomarkers in normal, MCI, and AD subjects diagnostic discrimination and cognitive correlations. Neurology 2009;73:287-293.

11 Toledo JB, Xie SX, Troganowski JQ, Shaw LM: Longitudinal change in CSF Tau and A $\beta$ biomarkers for up to 48 months in ADNI. Acta Neuropathol 2013;126:659-670.

12 Roberts RO, Knopman DS, Mielke MM, Cha RH, Pankratz VS, Christianson TJH, Geda YE, Boeve BF, Ivnik RJ, Tangalos EG: Higher risk of progression to dementia in mild cognitive impairment cases who revert to normal. Neurology 2014;82:317-325.

-13 Barnes DE, Cenzer IS, Yaffe K, Ritchie CS, Lee SJ: Alzheimer's Disease Neuroimaging Initiative: a point-based tool to predict conversion from mild cognitive impairment to probable Alzheimer's disease. Alzheimers Dement 2014;10:646-655.

14 Busse A, Hensel A, Gühne U, Angermeyer MC, Riedel-Heller SG: Mild cognitive impairment long-term course of four clinical subtypes. Neurology 2006;67:2176-2185.

15 Manly JJ, Tang MX, Schupf N, Stern Y, Vonsattel JPG, Mayeux R: Frequency and course of mild cognitive impairment in a multiethnic community. Ann Neurol 2008;63:494-506.

16 Jungwirth S, Zehetmayer S, Hinterberger M, Tragl KH, Fischer P: The validity of amnestic MCI and non-amnestic MCI at age 75 in the prediction of Alzheimer's dementia and vascular dementia. Int Psychogeriatr 2012;24: 959-966. 
17 Yaffe K, Petersen RC, Lindquist K, Kramer J, Miller B: Subtype of mild cognitive impairment and progression to dementia and death. Dement Geriatr Cogn Disord 2006;22:312-319.

18 Morris JC, Weintraub S, Chui HC, Cummings J, DeCarli C, Ferris S, Foster NL, Galasko D, Graff-Radford N, Peskind ER: The Uniform Data Set (UDS): clinical and cognitive variables and descriptive data from Alzheimer Disease Centers. Alzheimer Dis Assoc Disord 2006;20:210-216.

19 National Alzheimer's Coordinating Center: The UDS study population. https://www.alz.washington.edu/ WEB/study_pop.html (accessed September 27, 2016).

20 Flegal KM, Kit BK, Graubard BI: Body mass index categories in observational studies of weight and risk of death. Am J Epidemiol 2014;180:288-296.

-21 Pfeffer RI, Kurosaki TT, Harrah CH, Chance JM, Filos S: Measurement of functional activities in older adults in the community. J Gerontol 1982;37:323-329.

22 Cummings JL, Mega M, Gray K, Rosenberg-Thompson S, Carusi DA, Gornbein J: The Neuropsychiatric Inventory comprehensive assessment of psychopathology in dementia. Neurology 1994;44:2308-2308.

-23 Folstein MF, Folstein SE, McHugh PR: "Mini-mental state:" a practical method for grading the cognitive state of patients for the clinician. J Psychiatr Res 1975;12:189-198.

-24 Morris JC: Clinical dementia rating: a reliable and valid diagnostic and staging measure for dementia of the Alzheimer type. Int Psychogeriatr 1997;9:173-176.

25 Morris JC: The Clinical Dementia Rating (CDR): current version and scoring rules. Neurology 1993;43:24122414.

26 Fine JP, Gray RJ: A proportional hazards model for the subdistribution of a competing risk. J Am Stat Assoc 1999;94:496-509.

27 Lau B, Cole SR, Gange SJ: Competing risk regression models for epidemiologic data. Am J Epidemiol 2009;170: 244-256.

28 Grambsch PM, Therneau TM: Proportional hazards tests and diagnostics based on weighted residuals. Biometrika 1994;81:515-526.

29 Pankratz VS, Roberts RO, Mielke MM, Knopman DS, Jack CR, Geda YE, Rocca WA, Petersen RC: Predicting the risk of mild cognitive impairment in the Mayo Clinic Study of Aging. Neurology 2015;84:1433-1442.

-30 Knopman DS, Roberts RO, Geda YE, Boeve BF, Pankratz VS, Cha RH, Tangalos EG, Ivnik RJ, Petersen RC: Association of prior stroke with cognitive function and cognitive impairment: a population-based study. Arch Neurol 2009;66:614-619.

-31 Solfrizzi V, Panza F, Colacicco AM, D’Introno A, Capurso C, Torres F, Grigoletto F, Maggi S, Del Parigi A, Reiman EM: Vascular risk factors, incidence of MCI, and rates of progression to dementia. Neurology 2004;63:18821891.

-32 Amieva H, Letenneur L, Dartigues J-F, Rouch-Leroyer I, Sourgen C, D’Alchée-Birée F, Dib M, Barberger-Gateau P, Orgogozo J-M, Fabrigoule C: Annual rate and predictors of conversion to dementia in subjects presenting mild cognitive impairment criteria defined according to a population-based study. Dement Geriatr Cogn Disord 2004;18:87-93.

33 Jorm AF, Jolley D: The incidence of dementia. A meta-analysis. Neurology 1998;51:728-733.

34 Fleisher AS, Sowell BB, Taylor C, Gamst AC, Petersen RC, Thal LJ: Clinical predictors of progression to Alzheimer disease in amnestic mild cognitive impairment. Neurology 2007;68:1588-1595.

-35 Bretsky P, Guralnik JM, Launer L, Albert M, Seeman TE: The role of APOE- $\varepsilon 4$ in longitudinal cognitive decline MacArthur Studies of Successful Aging. Neurology 2003;60:1077-1081.

-36 Petersen RC, Smith GE, Ivnik RJ, Tangalos EG, Schaid DJ, Thibodeau SN, Kokmen E, Waring SC, Kurland LT: Apolipoprotein E status as a predictor of the development of Alzheimer's disease in memory-impaired individuals. JAMA 1995;273:1274-1278.

37 Mayeux R, Saunders AM, Shea S, Mirra S, Evans D, Roses AD, Hyman BT, Crain B, Tang M, Phelps CH: Utility of the apolipoprotein E genotype in the diagnosis of Alzheimer's disease. N Engl J Med 1998;338:506-511.

-38 Devanand DP, Pelton GH, Zamora D, Liu X, Tabert MH, Goodkind M, Scarmeas N, Braun I, Stern Y, Mayeux R: Predictive utility of apolipoprotein E genotype for Alzheimer disease in outpatients with mild cognitive impairment. Arch Neurol 2005;62:975-980.

39 Aggarwal NT, Wilson RS, Beck Todd L, Bienias JL, Berry-Kravis E, Bennett DA: The apolipoprotein E $\varepsilon 4$ allele and incident Alzheimer's disease in persons with mild cognitive impairment. Neurocase 2005;11:3-7.

40 Brodaty H, Seeher K, Gibson L: Dementia time to death: a systematic literature review on survival time and years of life lost in people with dementia. Int Psychogeriatr 2012;24:1034-1045.

-41 Alzheimer's Association: 2015 Alzheimer's disease facts and figures. Alzheimers Dement 2015;11:332. 